\title{
BOUNDARY VALUE PROBLEMS FOR VARIATIONAL INTEGRALS INVOLVING SURFACE CURVATURES
}

\author{
BY \\ JOHANNES C. C. NITSCHE \\ University of Minnesota, Minneapolis, Minnesota
}

\begin{abstract}
The following investigation deals with surfaces governed by and extremal for a free energy functional which is quadratic in the principal curvatures. The associated Euler-Lagrange differential equations are derived, as are the corresponding intricate natural boundary conditions. Pertinent boundary value problems-without and with volume constraints-are formulated and discussed ${ }^{1}$ and existence proofs are provided for certain situations. The discussion opens the view onto an arena of rich mathematical problems which will also be of interest in engineering applications where the surfaces in question are utilized frequently as idealized models for the interfaces separating phases in real materials.
\end{abstract}

1. Let $\mathscr{S}=\{\mathbf{x}=\mathbf{x}(u, v) ;(u, v) \in P\}$ be a differential geometric surface embedded in Euclidean space $R^{3}$. The position vector

$$
\mathbf{x}(u, v)=(x(u, v), y(u, v), z(u, v))
$$

is defined over a prescribed parameter domain $P-\mathrm{a}$ connected set with or without given boundaries. We wish to consider this surface not so much as a geometric object but as an idealized model for the interfaces or middle surfaces occurring in real materials-open or closed lipid bilayers and surfactant films, thin elastic plates, etc. $^{2}$ Thus, we associate with $\mathscr{S}$ a free energy $\hat{\Phi}$, per unit area, which incorporates surface tension but also reflects elastic properties. The latter involve the surface curvatures, and so $\hat{\Phi}$ will have the form $\hat{\Phi}=\hat{\Phi}\left(\kappa_{1}, \kappa_{2}\right)$. Here $\kappa_{1}=1 / R_{1}$ and $\kappa_{2}=1 / R_{2}$ denote the principal curvatures of $\mathscr{S}$. As a consequence, the total energy of $\mathscr{S}$ becomes

$$
\mathscr{E} \equiv \mathscr{E}(\mathscr{S})=\iint_{\mathscr{S}} \hat{\Phi}\left(\kappa_{1}, \kappa_{2}\right) d A .
$$

\footnotetext{
Received August 6, 1991.

1991 Mathematics Subject Classification. Primary 35J35, 35J40, 35J60, 35J65, 49Q05, 49Q10, 53A10, 73C50; Secondary 31B30, 31G15, 35P15, 35Q35, 35R35, 53A05, 53C42, 76B45.

${ }^{1}$ Some of the lengthier calculations are omitted.

${ }^{2}$ Realistically speaking, of course, the concept of sharp physical interfaces amenable to treatment with the tools of differential geometry is only an approximation, though often accurate and appropriate, to the actual narrow zones separating phases. For a discussion of the microstructure governing these zones in real materials, see, e.g. $[1,2,11,37,39,40,63,68]$.
} 
( $d A$ denotes the area element.) There may be further energy terms attributable to the boundary $\partial \mathscr{S}$ of $\mathscr{S}$. Two universal structural conditions will be imposed on the integrand in (1):

(1) $\hat{\Phi}\left(\kappa_{1}, \kappa_{2}\right)$ is a symmetric function of its arguments.

(2) $\mathscr{E}$ is definite in the following sense: There is a constant $c>-\infty$, possibly negative, such that $\mathscr{E}(\mathscr{T}) \geq c$ for every connected orientable surface $\mathscr{T}$ of regularity class $C^{2}$, with or without boundary. We allow the lower bound for $\mathscr{E}$ to be negative to reflect the fact that the free surface energy of an interface need not be positive; however, disregarding possible extraneous information concerning the magnitude of physical constants or the limiting influence of boundary conditions in specific applications (see, e.g., Sec. 10 and (34) below), we shall insist on the universal condition $c>-\infty$.

It should be stressed that our condition of definiteness is mathematical in nature; it does not take into account the structural properties of the admissible comparison surfaces $\mathscr{T}$ on the molecular level; see footnote 2 .

Under mild regularity assumptions (for a polynomial $\hat{\Phi}$, as a consequence of the fundamental theorem on symmetric polynomials), condition (1) above implies that $\hat{\Phi}$ can be written in the form $\hat{\Phi}\left(\kappa_{1}, \kappa_{2}\right)=\Phi(H, K)$, where $H=\left(\kappa_{1}+\kappa_{2}\right) / 2$ and $K=\kappa_{1} \kappa_{2}$ are the mean and Gaussian curvatures of $\mathscr{S}$, respectively. Moreover, rather than working with general nonlinear integrands, it is consistent with standard engineering practice, at least as a first step, that we limit consideration to the inclusion of terms up to those of second order, so that $\Phi$ becomes $\Phi(H, K)=a_{0}+a_{1} H+$ $a_{2} H^{2}+a_{3} K$. The second condition (2) of definiteness imposes specific restrictions on the coefficients $a_{0}, \ldots, a_{3}$. In fact, an analysis similar to that given on p. 14 of [55] shows that $\Phi$ must have the form

$$
\Phi(H, K)=\alpha+\beta\left(H-H_{0}\right)^{2}-\gamma K,
$$

with material constants (surface tension, elastic moduli) $\alpha, \beta, \gamma$ satisfying the conditions

$$
\alpha \geq 0, \quad 0 \leq \gamma \leq \beta, \quad \beta \gamma H_{0}^{2} \leq \alpha(\beta-\gamma) .
$$

It is the integrand (2), as well as the complex relations springing from it, which will be at the center of the discussion to follow. This integrand, or expressions closely resembling it-invariably including $\Phi=\left(\kappa_{1}+\kappa_{2}\right)^{2}=4 H^{2}, \Phi=\left(\kappa_{1}-\kappa_{2}\right)^{2}=$ $4\left(H^{2}-K\right)$, and $\Phi=\kappa_{1}^{2}+\kappa_{2}^{2}=4 H^{2}-2 K$-can be found in numerous sources from the earliest times (see, e.g., S. D. Poisson [59, pp. 221-225], [60, p. 321], S. Germain [21, pp. 12, 16], G. R. Kirchhoff [44, p. 63], F. Casorati [7, p. 109], A. E. H. Love $[48$, p. 130]) and has attracted renewed attention through the work of W. Helfrich [34] and fellow scientists (among others, [17, 28, 35, 37, 38, 58]; for further references also [54, Sec. 11]). The case $\Phi=1$ leads to the venerable theory of minimal surfaces (see $[49,51,54]$ ), and the case $\Phi=H^{2}$ - to be sure, in all investigations published so far exclusively restricted to closed surfaces, embedded or immersed (i.e., surfaces with possible self-intersections) - has been of considerable recent interest to geometers; references can be found in $[6,70,71,73,77]$ and [54, Sec. 11]. Generally speaking, there are today few mathematical treatments and no 
general existence theorems for the various physical boundary value problems arising in connection with (1), (2).

The "natural" (or "intrinsic", "spontaneous") curvature $H_{0}$ in (2) can often be related to the initial state of the interface or to the fact that the two sides of a bilayer may be chemically different; see, e.g., $[34,17,28]$. The actual numerical values of the constants $\alpha, \beta, \gamma$, and $H_{0}$ are of secondary importance at the moment. In many applications, $\alpha$ is larger by orders of magnitude than $\beta$ and $\gamma$, a fact which often lets us approach the interface $\mathscr{S}$ as the perturbation of an interface governed by the energy functional $\iint d A$ based on the integrand $\Phi \equiv 1$, i.e., a minimal surface (or a surface of constant mean curvature), depending on $\beta$ and $\gamma$ as control parameters. In other cases, for instance for menisci and vesicles with a size in the range of a few hundred $\AA$, the influence of $\alpha$ and that of $\beta, \gamma$ become comparable.

The case $\alpha=0, H_{0}=0, \beta=\gamma>0$, i.e., $\Phi=H^{2}-K$, is special. ${ }^{3}$ Here the associated energy $\mathscr{E}$ has the value zero for all spheres and spherical caps. Thus there may exist continua of solution surfaces for our Problem 1 below, and specific solution surfaces will have to be characterized by their area or by the volume they enclose.

2. One observation: The inclusion of terms of third order, that is, the addition of $a_{4} H^{3}+a_{5} H K$ to the expression $\Phi(H, K)$ in (2) above, leads to an indefinite energy functional unless $a_{4}=a_{5}=0$. This can be seen as follows. For thin cylinders for which $K=0$, the term $\iint_{\mathscr{S}} H^{3} d A$ can be made dominatingly negative so that the coefficient $a_{4}$ must be zero. As for the other part, consider as comparison surface the torus

$\mathscr{T}=\{x=(a+b \cos u) \cos v, y=(a+b \cos u) \sin v, z= \pm b \sin u ; 0 \leq u, v \leq 2 \pi\}$,

where $0<b<a$. Set $\lambda=b / a$. For this torus,

$$
\begin{gathered}
d A=b(a+b \cos u) d u d v, \quad H= \pm \frac{1}{2 b} \frac{a+2 b+\cos u}{a+b \cos u}, \\
K=\frac{1}{b} \frac{\cos u}{a+b \cos u},
\end{gathered}
$$

so that

$$
\begin{gathered}
\iint_{\mathscr{T}} d A=4 \pi^{2} a^{2} \lambda, \quad \iint_{\mathscr{T}} H d A= \pm 2 \pi^{2} a, \quad \iint_{\mathscr{T}} K d A=0, \\
\iint_{\mathscr{T}} H^{2} d A=\frac{\pi^{2}}{\lambda \sqrt{1-\lambda^{2}}}, \quad \iint_{\mathscr{T}} H K d A= \pm \frac{\pi^{2}}{a \sqrt{1-\lambda^{2}}\left(1+\sqrt{1-\lambda^{2}}\right)} .
\end{gathered}
$$

Obviously, the energy $\mathscr{E}(\mathscr{T})$ can be made as negative as desired for a suitable choice of the radii $a$ and $b$. Thus we must also have $a_{5}=0$.

It follows that the next step toward greater generality in the selection of the integrand $\hat{\Phi}\left(\kappa_{1}, \kappa_{2}\right)$ would have to include fourth powers of the principal curvatures.

\footnotetext{
${ }^{3}$ The combination $\left(H^{2}-K\right) d A$ is invariant under conformal transformations of $R^{3}$; see [8, 74]. The same is true for the differential equation (25); see [9].
} 
On the phenomenological level, there are suggestions of such integrands; see, e.g., [3, $17,37,38]$. On the mathematical level, the attending problems are formidable.

3. The static equilibrium shape of our interface $\mathscr{S}$ is determined by the condition that $\mathscr{S}$ be energy minimizing or, more generally and less restrictive, that $\mathscr{S}$ be an extremal for the energy functional $\mathscr{E}$, i.e., $\mathscr{S}$ must be a solution of the variational problem $\delta \mathscr{E}=0$. This variational problem leads not only to the fundamental Euler-Lagrange differential equation, but also to certain specific intrinsic, or natural, boundary conditions. For the actual determination of these, we have to investigate the change of the functional $\mathscr{E}$ associated with a variation of the surface $\mathscr{S}$. Given that the present discussion is not restricted to closed shapes (vesicles), as for instance the walls bounding blood cells, but includes surfaces with boundaries, fixed or free, the variations to be considered here cannot remain restricted to the special normal variations customarily employed in the literature.

The surface $\mathscr{S}$ will be regarded as the member of a one-parameter family of surfaces $\mathscr{S}(t)=\{\mathbf{x}=\mathbf{x}(u, v ; t) ;(u, v) \in P\},|t|<t_{0}$, such that $\mathbf{x}(u, v ; 0)=\mathbf{x}(u, v)$. We set $\mathbf{y}(u, v)=\mathbf{x}_{t}(u, v ; 0)$; then $\mathbf{x}(u, v ; t)=\mathbf{x}(u, v)+t \mathbf{y}(u, v)+O\left(t^{2}\right)$. A subsequent stability analysis would involve a discussion of the second variation $\delta^{2} \mathscr{E}$ and requires inclusion of the next term $\mathbf{x}_{t t}(u, v ; 0)$ in the expansion for $\mathbf{x}(u, v ; t)$. Note that the variation $\mathbf{y}(u, v)$ has a component $\mathbf{y}-(\mathbf{y} \cdot \mathbf{X}) \mathbf{X}$ tangent to $\mathscr{S}$ and a component $\mathbf{y}(u, v) \cdot \mathbf{X}(u, v)$ normal to $\mathscr{S}$. Here $\mathbf{X}(u, v)=(X(u, v), Y(u, v), Z(u, v))$ is the unit normal vector.

For any functional $\mathscr{F} \equiv \mathscr{F}(\mathscr{S})$, we shall use the standard abbreviation

$$
\delta \mathscr{F}=\left.\frac{\partial}{\partial t} \mathscr{F}(\mathscr{S}(t))\right|_{t=0} .
$$

A rather lengthy computation leads to the following variation formulae:

$$
\begin{gathered}
\delta \iint_{\mathscr{S}} d A=-2 \iint_{\mathscr{S}} H(\mathbf{y} \cdot \mathbf{X}) d A-\int_{\partial \mathscr{S}}(\mathbf{y}, \mathbf{X}, d \mathbf{x}) \\
\delta \iint_{\mathscr{S}} \Psi(H) d A= \\
\iint_{\mathscr{S}}\left\{\Delta \Psi_{H}+2\left(2 H^{2}-K\right) \Psi_{H}-4 H \Psi\right\}(\mathbf{y} \cdot \mathbf{X}) d A \\
-\int_{\partial \mathscr{S}} \Psi(\mathbf{y}, \mathbf{X}, d \mathbf{x})+\frac{1}{2} \int_{\partial \mathscr{S}}\left\{\Psi_{H} \frac{\partial(\mathbf{y} \cdot \mathbf{X})}{\partial n}-(\mathbf{y} \cdot \mathbf{X}) \frac{\partial \Psi_{H}}{\partial n}\right\} d s \\
\delta \iint_{\mathscr{S}} K d A=-\int_{\partial \mathscr{S}} K(\mathbf{y}, \mathbf{X}, d \mathbf{x})+\int_{\partial \mathscr{P}}\left\{\Lambda^{(1)} \frac{\partial(\mathbf{y} \cdot \mathbf{X})}{\partial n}-\Lambda^{(2)}(\mathbf{y} \cdot \mathbf{X})\right\} d s
\end{gathered}
$$

For our present purposes, the role of the expression $\Psi(H)$ will be taken by $\left(H-H_{0}\right)^{2}$. Of course, $\Delta \Psi_{H}=\Psi_{H H} \Delta H+\Psi_{H H H} \nabla(H, H)$. Here $\Psi_{H}=\partial \Psi / \partial H$ etc., and $\nabla, \Delta$ represent the first and second Beltrami operator on $\mathscr{S}$. If the surface $\mathscr{S}$ is before us in a nonparametric representation $z=z(x, y)$ then the curvatures of $\mathscr{S}$ 
take the form $\left(p=z_{x}, q=z_{y}, r=z_{x x}, s=z_{x y}, t=z_{y y}, W=\sqrt{1+p^{2}+q^{2}}\right)$

$$
\begin{gathered}
H=\frac{\left(1+q^{2}\right) r-2 p q s+\left(1+p^{2}\right) t}{2 W^{3}}, \\
K=\frac{r t-s^{2}}{W^{4}} .
\end{gathered}
$$

Further, for functions $f(x, y), g(x, y)$ defined on $\mathscr{S}$, we have

$$
\begin{array}{r}
\boldsymbol{\nabla}(f, g)=\frac{1}{W^{2}}\left\{\left(1+q^{2}\right) f_{x} g_{x}-p q\left(f_{x} g_{y}+f_{y} g_{x}\right)+\left(1+p^{2}\right) f_{y} g_{y}\right\}, \\
\Delta f=\frac{1}{W}\left\{\frac{\partial}{\partial x}\left(\frac{\left(1+q^{2}\right) f_{x}-p q f_{y}}{W}\right)+\frac{\partial}{\partial y}\left(\frac{-p q f_{x}+\left(1+p^{2}\right) f_{y}}{W}\right)\right\} \\
=\frac{1}{W^{2}}\left\{\left(1+q^{2}\right) f_{x x}-2 p q f_{x y}+\left(1+p^{2}\right) f_{y y}\right\}-\frac{2 H}{W}\left(p f_{x}+q f_{y}\right) .
\end{array}
$$

Observe that $\Delta x=-2 p H / W, \Delta y=-2 q H / W$, as well as

$$
\Delta z=\frac{2 H}{W} \text {. }
$$

This is the nonparametric equivalent of the general relation

$$
\Delta \mathbf{x}=2 H \mathbf{X} .
$$

4. If $\mathscr{S}$ is a closed surface, then no boundary terms appear in the variational equations (7), (8), and (9). In general, however, such terms will be present and will have to be contended with. Therefore, a number of explanations as well as, in particular, a geometric interpretation of the expressions $\Lambda^{(1)}$ and $\Lambda^{(2)}$ on the right-hand side of (9) are called for. ${ }^{4}$

For any variation $\mathbf{y}$ parallel to the surface normal vector $\mathbf{X}$, the triple product $(\mathbf{y}, \mathbf{X}, d \mathbf{x})$ is zero. Concerning the boundary terms, note that the boundary $\partial \mathscr{S}$ consists of one or several contours. Each of these contours has the double existence as a curve in $R^{3}$ and as a curve on $\mathscr{S}$. We denote by $k$ and $\tau$ the space curvature and the torsion, respectively, of this curve and by $k_{n}$ and $k_{g}$ its normal and geodesic curvatures. Of course, $k^{2}=k_{n}^{2}+k_{g}^{2}$. With the help of these quantities, a computation shows that the expressions $\Lambda^{(1)}, \Lambda^{(2)}$ take the form

$$
\begin{aligned}
& \Lambda^{(1)}=k_{n}, \\
& \Lambda^{(2)}=\frac{\partial \tau}{\partial s}+\frac{\partial}{\partial s}\left(\frac{k_{n}}{k^{2}} \frac{\partial k_{g}}{\partial s}\right)-\frac{\partial}{\partial s}\left(\frac{k_{g}}{k^{2}} \frac{\partial k_{n}}{\partial s}\right) .
\end{aligned}
$$

Generally, the symbols $\partial / \partial s$ and $\partial / \partial n$ indicate tangential differentiations along $\partial \mathscr{S}$ and normal to $\partial \mathscr{S}$, respectively, according to the metric on $\mathscr{S}$. If we denote

\footnotetext{
${ }^{4}$ In the various engineering applications, a physical interpretation of these expressions is of importance as well. See also footnote 5 .
} 
by $\vartheta$ the angle between the principal normal of $\partial \mathscr{S}$ and the normal $\mathbf{X}$ of $\mathscr{S}$, then (17) can also be written in the simpler form

$$
\Lambda^{(2)}=\frac{\partial \tau}{\partial s}+\frac{\partial^{2} \vartheta}{\partial s^{2}} .
$$

Relations (17) or (18) are ambiguous for straight parts of the boundary $\partial \mathscr{S}$ where $k=k_{n}=k_{g}=\tau=0$. Along these parts, they become

$$
\Lambda^{(2)}=\frac{\partial^{2} \vartheta}{\partial s^{2}},
$$

where now $\vartheta$ is to be taken as the angle which the surface normal $\mathbf{X}$ forms with a chosen fixed direction.

5. In view of the arbitrary character of the variation $\mathbf{y}(u, v)$, standard arguments of the calculus of variations lead to the Euler-Lagrange equation of our variational problem:

$$
\beta\left\{\Delta H+2\left(H-H_{0}\right)\left(H^{2}-K+H H_{0}\right)\right\}-2 \alpha H=0,
$$

or equivalently,

$$
\beta\left\{\Delta H+2 H\left(H^{2}-K\right)\right\}-2\left(\alpha+\beta H_{0}^{2}\right) H+2 \beta H_{0} K=0 .
$$

It must be understood that solution surfaces of this differential equation need not be energy minimizing. (21) is only a necessary condition; in fact, it does not even contain the coefficient $\gamma$. As a consequence, the differential equation (21), by itself, generally can provide no clue regarding the energy level $\mathscr{E}(\mathscr{S})$ of its solution surfaces. To wit, by the Gauss-Bonnet theorem, the integral $\iint_{\mathscr{S}} K d A$ has the same value $4 \pi(1-g)$ for all closed surfaces of the same genus $g$ ( $g=0$ for a sphere, $g=$ 1 for a torus etc.). Thus $\delta \iint_{\mathscr{S}} K d A=0$ for any closed surface, so that (21) clearly will contain no contribution stemming from the term $-\gamma K$ in (2). The significant fact that the coefficient $\gamma$ is absent in (21) also for surfaces with boundaries is, of course, a discovery, of S. D. Poisson, more than 175 years ago and predating the Gauss-Bonnet thoeorem; see [59, pp. 224-225], also [54, Sec. 11], [55, p. 16]. The term $-\gamma K$ is nevertheless of consequence. As is apparent from an inspection of (22), (23) below, it manifests itself in the boundary conditions and, of course even more so, in the expression of the second variation. Thus, this term does influence the value of the energy, particularly in the case of "crumpled" boundaries. ${ }^{5}$

The natural boundary conditions accompanying (21) can be obtained by grouping the coefficients of the variational components $(\mathbf{y} \cdot \mathbf{X}), \partial(\mathbf{y} \cdot \mathbf{X}) / \partial n$, and $(\mathbf{y}, \mathbf{X}, d \mathbf{x})$. This leads to the following groups, in the same order:

$$
\begin{gathered}
-\beta \frac{\partial H}{\partial n}-\gamma \Lambda^{(2)}, \\
\beta\left(H-H_{0}\right)-\gamma k_{n}, \\
-\alpha-\beta\left(H-H_{0}\right)^{2}+\gamma K .
\end{gathered}
$$

\footnotetext{
${ }^{5}$ In connection with a discussion of lipid-water systems, S. M. Gruner [28, p. 7570] states an open question: "The significance of the Gaussian term...is unclear. It is not known whether this term modulates the phase behavior. The value of $k_{g}$ [related to our $-\gamma$ ] is not known."
} 
The question, which of these expressions must be equated to zero, depends on the concrete problem before us. As for (24), it is possible that further contributions to the energy functional attributable to the fixed boundaries must be considered. We shall not pursue this here; see, however, Sec. 14 below.

6. Equation (21) is a nonlinear partial differential equation of fourth order for the position vector of $\mathscr{S}$. It is a fortunate circumstance that this differential equation can be written in the form of four differential equations of second order-three, namely (15), for the components of the position vector $\mathbf{x}$, and one, namely (21), for the mean curvature $H$. For the case of a surface in nonparametric representation $z=z(x, y)$, these equations reduce to two coupled highly nonlinear elliptic equations of second order. The expressions in (22), (23), (24) establish the relation between the position vector and the mean curvature of $\mathscr{S}$ on the boundary $\partial \mathscr{S}$ and provide for the appropriate boundary conditions.

We note that the case of the special integrand $\Phi(H, K)=H^{2}$ leads to the differential equation

$$
\Delta H+2 H\left(H^{2}-K\right)=0 .
$$

In nonparametric form, this differential equation was derived by S. D. Poisson [ 59 , p. 224]. Its invariant form is due to W. Schadow; see [72, p. 56].

7. Often the interface separates two media of prescribed volumes. We denote by $\mathscr{V}(\mathscr{S})$ one of these volumes. The admissible variations of $\mathscr{S}$ are now subject to the volume constraint $\mathscr{V}(\mathscr{S})=$ const, and our variational problem becomes

$$
\delta \iint_{\mathscr{S}} \phi(H, K) d A+\mu \delta \mathscr{V}(\mathscr{S})=0
$$

where $\mu$ is a Lagrange multiplier. A computation shows that

$$
\delta \mathscr{V}=-\iint_{\mathscr{S}}(\mathbf{y} \cdot \mathbf{X}) d A-\int_{\partial \mathscr{S}}(\mathbf{y}, \mathbf{Q}(\mathbf{x}), d \mathbf{x}) .
$$

For a description of the vector function $\mathbf{Q}(\mathbf{x})$, see [52] and [53, p. 5]. Assume in particular that the boundary $\partial \mathscr{S}$ is required to lie on a prescribed surface; say, assume that $\mathscr{S}$ spans the interior of a region $\mathscr{B}$ in $R^{3}$ with fixed boundary $\partial \mathscr{B}$. Then the vector function has the property that $\mathbf{Q}(\mathbf{x})$ is tangent to this boundary at each point $\mathbf{x} \in \partial \mathscr{B}$.

In the same context, it is possible that the total energy of the system under consideration contains a contribution that is proportional to the area of $\partial \mathscr{B}$ wetted by one of the phases. Let us describe this area by a functional $\mathscr{W}(\mathscr{S})$. The change of $\mathscr{W}$ under a variation of $\mathscr{S}$ comes to

$$
\delta \mathscr{W}=\iint_{\partial \mathscr{S}}(\mathbf{y}, \mathbf{N}(\mathbf{x}), d \mathbf{x}) .
$$

Here $\mathbf{N}(\mathbf{x})$ denotes the unit normal vector of $\partial \mathscr{B}$ at the point $\mathbf{x}$. The derivation of the Euler-Lagrange equations involves yet another multiplier $\nu$ :

$$
\delta\{\mathscr{E}(\mathscr{S})+\mu \mathscr{V}(\mathscr{S})+\nu \mathscr{W}(\mathscr{S})\}=0 .
$$


In the presence of the functionals $\mathscr{V}$ and $\mathscr{W}$, the differential equation (21) has to be complemented, and it will ultimately take the form

$$
\beta\left\{\Delta H+2 H\left(H^{2}-K\right)\right\}-2\left(\alpha+\beta H_{0}^{2}\right) H+2 \beta H_{0} K-\mu=0 .
$$

Obviously, the boundary conditions will have to be complemented as well. The multiplier $\nu$, which does not enter (30) explicitly, will appear in the new conditions.

8. While there are extensive numerical investigations of the solution surfaces of (21) and (30), albeit, it must be said, generally restricted to surfaces with rotational symmetry (a stipulation which reduces the problem to one of ordinary differential equations!), very few analytical solutions are known today. As far as closed surfaces are concerned, we have of course the spheres. Moreover, an inspection of (5), in conjunction with the relations

$$
\Delta H=\frac{1}{b(a+b \cos u)} \frac{\partial}{\partial u}\left(\frac{a+b \cos u}{b} \frac{\partial H}{\partial u}\right)=-\frac{a}{2 b^{2}} \frac{b+a \cos u}{(a+b \cos u)^{3}}
$$

and

$$
H^{2}-K=\frac{a^{2}}{4 b^{2}(a+b \cos u)^{2}},
$$

shows that the torus $\mathscr{T}$ of (4) satisfies the differential equation (30) for the following choice of the coefficients:

$$
a=\sqrt{2} b, \quad b=\frac{2 \beta H_{0}}{\alpha+\beta H_{0}^{2}}, \quad \mu=-\frac{\left(\alpha+\beta H_{0}^{2}\right)^{2}}{2 \beta H_{0}},
$$

if $H_{0} \neq 0$; see [57]. The case $H_{0}=0$ is possible only if $\alpha=0$ in (2); then also the Lagrange multipler $\mu$ must be zero and we are led from (30) to (25). The fact that an "anchor ring", i.e., the torus (4) whose generating circles have radii in the ratio $b: a=1: \sqrt{2}$, solves the differential equation (25), is a discovery of $\mathrm{B}$. Y. Chen; see, e.g., [73, p. 18]. Of course, $(25)$ is satisfied by all minimal surfaces. On the other hand, the anchor ring is the image under stereographic projection in an equatorial $R^{3}$ of the "Clifford torus" $\left\{x_{1}+i x_{2}=(1 / \sqrt{2}) e^{i \alpha}, x_{3}+i x_{4}=(1 / \sqrt{2}) e^{i \beta}\right.$; $0 \leq \alpha, \beta \leq 2 \pi\}$ lying in the standard sphere $S^{3}: x_{1}^{2}+x_{2}^{2}+x_{3}^{2}+x_{4}^{2}=1$. There is an intriguing relation between minimal surfaces in $S^{3}$ and solution surfaces of (25) in $R^{3}$. For details see, e.g., [43, 47, 71, 73, 77] and [54, Sec. 11].

9. The shapes of physical interest include closed surfaces, periodic surfaces, but also surfaces with boundaries required to satisfy appropriate boundary conditions. In view of their crystallographic symmetry properties, periodic solution surfaces can often be obtained by repeated reflections from specific surface patches. The latter appear as solutions of $(21)$ or $(30)$ whose position vector is defined over a polygonal domain and subject to specific boundary conditions. [55] contains an existence proof of periodic solution surfaces for the variational problem $\delta \mathscr{E}+\mu \delta \mathscr{V}=0$ in certain situations. Regarding the case $\Phi(H, K)=1$, i.e., periodic minimal surfaces and periodic surfaces of constant mean curvature, see $[16,41,42,56,65,66]$.

In the present investigation, we are mainly interested in fixed and free boundary value problems, that is, in the determination of minimizing or stationary surfaces 
for the energy functional, with boundaries on fixed curves or surfaces-the shape assumed by a fluid membrane closing the opening of a glass tube when pressure is applied in the tube, the shape of a film spanning a wire, etc. Two problems are representative:

Problem 1. Given a collection of disjoint simple closed curves $\Gamma_{1}, \ldots, \Gamma_{m}$ in $R^{3}$. To determine a surface $\mathscr{S}$ bounded by these curves which minimizes, or makes stationary, the value of the energy $\mathscr{E}$ in the class of all surfaces of a prescribed topological type,

(a) not subject to a volume constraint (differential equation (21));

(b) subject to a volume constraint (differential equation (30)).

Problem 1 can be considered as a generalization of the Plateau-Douglas problem which is based on the integrand $\Phi(H, K) \equiv 1$, i.e., $\alpha>0, \beta=\gamma=0$, and which leads to minimal surfaces and to surfaces of constant mean curvature. For this case, traditionally the most effective approach to the existence problems for energy minimizing (here: area minimizing) surfaces utilizes the direct method of the calculus of variations. A similar, albeit by necessity far more complex approach, should turn out to be useful also in the present context. As is well known, however, the existence proof for extremal surfaces which are merely stationary requires additional arguments. Since the condition $H=0$ for minimal surfaces can be interpreted as an elliptic differential equation for the position vector of the desired surface-a very special case of the differential equations (15), (21) - the direct methods may be complemented by methods from the theory of partial differential equations, a circumstance that is particularly relevant for surfaces in a nonparametric representation $z=z(x, y)$. In all discussions to follow, we assume that $\beta>0$.

One of the most interesting special cases for Problem 1 is that of a single fixed curve $\Gamma$ bounding simply connected surfaces, i.e., surfaces of disc-type.

Problem 2. Given a fixed surface in $R^{3}$. To determine an interface $\mathscr{S}$ of least, or stationary, energy with boundary lying on this surface,

(a) not subject to a volume constraint (differential equation (21));

(b) subject to a volume constraint (differential equation (30)).

A typical example of Problem 2 has been described in Sec. 7 above; for a further discussion, see Sec. 14.

Finally, a combination of Problems 1 and 2 leads to the determination of surfaces whose boundaries are partially fixed and partially free, in the spirit of [51, VI.2]. We have neglected here consideration of further forces and moments on the fixed and the supporting boundaries of $\mathscr{S}$ which would lead to a modification of the problems.

It is clear that Problems 1 and 2 require a discussion concerning the regularity of the potential solution surfaces. Such a discussion has not yet been attempted. For surfaces that are stationary, not necessarily minimizing, with respect to the area functional (the special case $\alpha>0, \beta=\gamma=0$ ), see [51, V.2.1, VI.2], [54, V.2.1], and $[13,29,30]$. If the bounding configuration has corners or edges, as will be the case in actual experimental situations, then also the local behavior of the solution surfaces near these boundary singularities needs to be elucidated. For surfaces in a nonparametric representation $z=z(x, y)$; see [27, 46, 75, 76]. As for surfaces in 
a parametric representation, an exhaustive discussion is so far available only for the special case $\beta=\gamma=0$; see [54, Secs. 357-360, 382], [12, 33, 36, 66, 67].

10. Let us turn to Problem 1 in its simplest form, that of one contour $\Gamma$ bounding a surface of the type of the circular disc. Assume that $\Gamma$ is given in a representation $\Gamma=\{\mathbf{x}=\mathbf{z}(s) ; 0 \leq s \leq L\}$ in terms of its arc length $s$. We denote the tangent, principal normal and binormal of $\Gamma$ by $\mathbf{t}, \mathbf{n}$, and $\mathbf{b}$, respectively, and the curvature and torsion by $k$ and $\tau$. A potential solution surface may be before us in a representation $\mathscr{S}=\{\mathbf{x}=\mathbf{x}(u, v) ;(u, v) \in \bar{P}\}$. Here $\bar{P}$ denotes the closure of the unit disc $P: u^{2}+v^{2}<1$ in the $(u, v)$-plane. $\mathscr{S}$ must satisfy the differential equation (21). As for the conditions on the boundary $\partial P$, note that all admissible comparison surfaces are required to have the same boundary curve, namely $\Gamma$. Then the variation vector $\mathbf{y}$ must be parallel to $d \mathbf{x}$ in the points of $\partial P$, in particular $\mathbf{y}(u, v) \cdot \mathbf{X}(u, v)=0$ for $(u, v) \in \partial P$. On the other hand, however, no restrictions can be placed on the values of $\partial(\mathbf{y} \cdot \mathbf{X}) / \partial n$ on the boundary of $P$. (It is true that for a clamped plate also this quantity would be zero.) As a consequence, in view of the remarks at the end of Sec. 4 , we find that we must have

$$
\beta\left(H-H_{0}\right)-\gamma k_{n}=0
$$

for $(u, v) \in \partial P$. Here $k_{n}=k(\mathbf{X} \cdot \mathbf{n})=\mathbf{X} \cdot(d \mathbf{t} / d s)$ is the normal curvature of $\Gamma$ considered as a curve on $\mathscr{S}$. If the surface $\mathscr{S}$ is known to us, then this normal curvature can be computed, and (32) can be seen as a condition on the mean curvature $H$ of $\mathscr{S}$. This is the requisite boundary condition for the partial differential equation (21).

The following observation is in order. By the Gauss-Bonnet theorem, we have

$$
\int_{\partial \mathscr{S}} k_{g} d s+\iint_{\mathscr{S}} K d A=2 \pi
$$

Thus, even though the value of $k_{g}=k(\mathbf{b} \cdot \mathbf{X})$ is not known, it follows from $\left|k_{g}\right| \leq k$ that

$$
-k(\Gamma)-2 \pi \leq-\iint_{\mathscr{S}} K d A \leq k(\Gamma)-2 \pi
$$

for any disc-type surface $\mathscr{S}$ bounded by the curve $\Gamma$. Here $k(\Gamma)=\int_{\Gamma} k d s$ is the total curvature of $\Gamma$. By W. Fenchel's theorem [14], $k(\Gamma) \geq 2 \pi$ and $k(\Gamma)=2 \pi$ only for plane convex curves. Since any minimal surface bounded by $\Gamma$ is a comparison surface for our variational problem, (34) implies the following a priori estimate for the value of the minimal energy for Problem la

$$
\mathscr{E}_{\min } \leq \alpha A(\Gamma)+\gamma\{k(\Gamma)-2 \pi\} .
$$

In this inequality, $A(\Gamma)$ stands for the greatest lower bound of the areas of all disctype surfaces bounded by $\Gamma$ (a property of $\Gamma$ alone). At the same time, we have the universal estimate

$$
\mathscr{E}(\mathscr{S}) \geq \alpha A(\mathscr{S})-\gamma\{k(\Gamma)+2 \pi\},
$$

and, of course, there may well be stationary solution surfaces with an energy level higher than $\mathscr{E}_{\min }$. 
In the application of the Gauss-Bonnet theorem above, it has been tacitly assumed that the surfaces $\mathscr{S}$ under consideration are differential geometrically regular. Even for the special case of minimal surfaces $(\beta=\gamma=0)$, this is assumed a priori only for the energy (= area) minimizing solutions. For minimal surfaces with isolated singularities, equation (33) must be replaced by relation (156) of [54].

11. It will be of interest to determine the specific form of the boundary condition (32) for the case of a surface $\mathscr{S}$ in a representation $z=z(x, y)$. Let us assume that the function $z(x, y)$, required to satisfy appropriate regularity conditions, is defined on the closure $\bar{D}$ of a domain $D$ in the $(x, y)$-plane. $D$ may be simply connected or have higher connectivity. Without loss of generality, we shall consider the former case.

The boundary $\partial D$ is given in the form $x=x(\sigma), y=y(\sigma)$ in terms of the arc length $\sigma$, and $\kappa=\kappa(\sigma)=x^{\prime}(\sigma) y^{\prime \prime}(\sigma)-x^{\prime \prime}(\sigma) y^{\prime}(\sigma)$ is its curvature. The differential geometric relations $x^{\prime \prime}=-\kappa y^{\prime}, y^{\prime}=\kappa x^{\prime}$ are valid. For Problem 1, the values of $z(x, y)$ are prescribed on $\partial D$ and will be denoted by $\varphi=\varphi(\sigma)$ so that $z(x(\sigma), y(\sigma))=\varphi(\sigma)$. The contour $\Gamma$ bounding $\mathscr{S}$ has the coordinates $x(\sigma), y(\sigma), \varphi(\sigma)$. Of course, $\sigma$ is not the arc length for $\Gamma$. The unit normal vector of $\mathscr{S}$ is $\left(-z_{x} / W,-z_{y} / W, 1 / W\right)$. Here $W=\sqrt{1+z_{x}^{2}+z_{y}^{2}}$. For points on $\partial D$, we can write $z_{x}^{2}+z_{y}^{2}=(\partial z / \partial \sigma)^{2}+(\partial z / \partial \nu)^{2}$, where $\partial z / \partial \nu$ denotes differentiation of $z$ with respect to the outer normal of $D$. Substitution into the expression of $k_{n}$ gives

$$
k_{n}=\frac{1}{1+\varphi^{\prime 2}} \frac{\varphi^{\prime \prime}+\kappa \partial z / \partial \nu}{\sqrt{1+\varphi^{\prime 2}+(\partial z / \partial \nu)^{2}}}
$$

In its complete form, then, Problem 1 comprises the following: The differential equations (14), (21) (for Problem 1(a)) or (14), (30) (for Problem 1(b)), to be satisfied in the domain $D$, along with the conditions

$$
\begin{gathered}
z=\varphi, \\
\beta\left(H-H_{0}\right)=\gamma \frac{1}{1+\varphi^{\prime 2}} \frac{\varphi^{\prime \prime}+\kappa \partial z / \partial \nu}{\sqrt{1+\varphi^{\prime 2}+(\partial z / \partial \nu)^{2}}},
\end{gathered}
$$

to be satisfied on the boundary $\partial D$. For both differential equations, these are Dirichlet conditions. (Recall that the structural requirements imposed on $\Phi(H, K)$ include the inequality $0 \leq \gamma \leq \beta$ according to which $\beta=0$ implies $\gamma=0$.)

If we restrict the last condition (39) to the terms of lowest order, thereby neglecting the contributions of higher powers of $\varphi$ and its derivatives as well as those of $z, z_{x}, \ldots, z_{y y}, H, H_{x}, \ldots, H_{y y}$, and if we set $H_{0}=0$, then (39) reduces to

$$
\beta H=\gamma\left(\varphi^{\prime \prime}+\kappa \frac{\partial z}{\partial \nu}\right)
$$


This relation can be found in the literature concerning the elastic theory of thin plates; see especially [18, pp. 600-605], further [44, pp. 68-69], [61, 62, 64]. It is obtained as a consequence of the customary approach approximating the quantities $d A, H$, and $K$ in the energy functional by the expressions $d x d y,\left(z_{x x}+z_{y y}\right) / 2$, and $z_{x x} z_{y y}-z_{x y}^{2}$. As is well known, at this level of approximation, the differential equations (14), (21), (30) become linear with a principal part which is effectively the biharmonic operator $\Delta^{2} z \equiv \Delta \Delta z \quad(\Delta$ denotes the Laplace operator). The boundary conditions generally favored in the literature are $z=\partial z / \partial \nu=0$; for these, uniqueness is guaranteed in all cases; regarding existence, see e.g. [4]. The boundary conditions (38), (40) are more intricate, and the question of nonuniqueness becomes pertinent.

The unabridged nonlinear theory is of course more complex. It should be noted, however, that the correct problem (14), (21) or (30), (38), (39) can be solved completely in many situations provided the function $\varphi(\sigma)$ and the spontaneous curvature $H_{0}$ are sufficiently small. Assume that the boundary $\partial D$ of the domain of definition belongs to class $C^{4, \lambda}$, where the Hölder exponent $\lambda$ is a number in the interval $0<\lambda<1$, and that the function $\varphi(\sigma)$ is of the same regularity class.

Theorem 1A. Assume that the quotient $\gamma / \beta$ is different from the eigenvalues of the problem (53)-(56) below-for a disk $D$, that $\alpha^{2}+H_{0}^{2}+(\beta-\gamma)^{2}>0$. There is a positive constant $m$ depending only on the domain $D$, on the material parameters $\alpha, \beta, \gamma$ and on a bound for $H_{0}$ with the following property. If $\|\varphi\|_{4, \lambda}^{\partial D}<m$ and $\left|H_{0}\right|<m$, then the problem (14), (21), (38), (39) has a unique solution $z(x, y)$ in the regularity class $C^{4, \lambda}(\bar{D})$ satisfying an inequality

$$
\|z\|_{4, \lambda}^{\bar{D}} \leq \mathscr{C}\left\{\|\varphi\|_{4, \lambda}^{\partial D}+\left|H_{0}\right|\right\} .
$$

Here the bound $\mathscr{C}$ depends on $m, \alpha, \beta, \gamma$ and on the properties of $D$, but is independent of $\varphi$ and $H_{0}$.

The norms in Theorem $1 \mathrm{~A}$ are the usual Schauder norms.

As already mentioned in Sec. 5, our boundary value problem is decomposed into two Dirichlet problems for a coupled system of second-order elliptic equations, namely (42), (38) for the function $z(x, y)$ and (43), (39) for the function $H(x, y)$. The coupling is strong because the functions $z, H$ and their derivatives appear not only in the right-hand sides of the differential equations and one of the boundary conditions, but $z$ also determines the principal part of the differential operators. The first problem (42), (38) is of course the classical Dirichlet problem for surfaces having prescribed mean curvature. It is well known that solvability and nonsolvability of this problem lead to intricate questions and depend on specific conditions; for details see $[5,15,19,20,22,23,24,25,26,31,32,54,69]$. Given this situation, it is a fair expectation that the general boundary value problems before us here will pose many highly interesting new challenges.

In preparation for the proof of Theorem $1 \mathrm{~A}$, we shall write the differential equations and the boundary conditions for $z$ and $H$ in the form 


$$
\begin{gathered}
\Delta z-2 H=f\left(z_{x}, z_{y}, z_{x x}, z_{x y}, z_{y y}, H\right) \\
\Delta H-2\left(\frac{\alpha}{\beta}+H_{0}^{2}\right) H=g\left(z_{x}, z_{y}, z_{x x}, z_{x y}, z_{y y}, H, H_{x}, H_{y}, H_{x x}, H_{x y}, H_{y y}\right) \\
z=\varphi \\
\beta H-\gamma \kappa(\sigma) \frac{\partial z}{\partial \nu}=\beta H_{0}+\gamma \varphi^{\prime \prime}+h\left(\varphi^{\prime}, \varphi^{\prime \prime}, \frac{\partial z}{\partial \nu}\right)
\end{gathered}
$$

where

$$
\begin{gathered}
f=2 H\left(W^{3}-1\right)-z_{y}^{2} z_{x x}+2 z_{x} z_{y} z_{x y}-z_{x}^{2} z_{y y} \\
g=2 W\left(z_{x} H H_{x}+z_{y} H H_{y}\right)-2 W^{2} H^{3}+2\left(H-H_{0}\right) \frac{z_{x x} z_{y y}-z_{x y}^{2}}{W^{2}} \\
-z_{y}^{2} H_{x x}+2 z_{x} z_{y} H_{x y}-z_{x}^{2} H_{y y}+2\left(\frac{\alpha}{\gamma}+H_{0}^{2}\right) H\left(W^{2}-1\right) \\
h=\gamma\left(\varphi^{\prime \prime}+\kappa \frac{\partial z}{\partial \nu}\right)\left(\frac{1}{1+\varphi^{\prime 2}} \frac{1}{\sqrt{1+\varphi^{\prime 2}+(\partial z / \partial \nu)^{2}}}-1\right)
\end{gathered}
$$

Here $W=\sqrt{1+z_{x}^{2}+z_{y}^{2}}$, as before, and $\Delta$ denotes the Laplace operator.

Note that the individual terms occurring in the right-hand sides $f, g, h$ in (42), (43), (45) are of second and higher degree in their respective arguments. We shall designate these right-hand sides also by the abbreviated notation $f=f(z, H)$, $g=g(z, H), h=h(\varphi, z)$.

Let us denote by $z^{(0)}=z^{(0)}(x, y)$ and $H^{(0)}=H^{(0)}(x, y)$ the solution pair of the linearized coupled system of Dirichlet problems,

$$
\begin{gathered}
\Delta z-2 H=0, \quad(x, y) \in D, \\
z=\varphi, \quad(x, y) \in \partial D,
\end{gathered}
$$

and

$$
\begin{gathered}
\Delta H-2\left(\frac{\alpha}{\beta}+H_{0}^{2}\right) H=0, \quad(x, y) \in D, \\
\beta H-\gamma \kappa(\sigma) \frac{\partial z}{\partial \nu}=\beta H_{0}+\gamma \varphi^{\prime \prime}, \quad(x, y) \in \partial D .
\end{gathered}
$$

12. It is important to establish that this solution pair is unique. For this, we have to investigate the homogeneous linearized eigenvalue problem

$$
\begin{aligned}
\Delta z-2 H & =0, & & (x, y) \in D, \\
\Delta H-c^{2} H & =0, & & (x, y) \in D, \\
z & =0, & & (x, y) \in \partial D, \\
H-\xi \kappa(\sigma) \frac{\partial z}{\partial \nu} & =0, & & (x, y) \in \partial D .
\end{aligned}
$$


Here $c^{2}=2\left(\alpha+\beta H_{0}^{2}\right) / \beta \geq 0$ and the constant $\xi$ replaces the quotient $\gamma / \beta$.

Integration of the difference $H \Delta z-z \Delta H$ leads to the relation

$$
\iint_{D}\left\{4 H^{2}+c^{2}\left(z_{x}^{2}+z_{y}^{2}\right)\right\} d x d y=2 \xi \int_{\partial D} \kappa(\sigma)\left(\frac{\partial z}{\partial \nu}\right)^{2} d \sigma
$$

which shows that the eigenvalues $\xi$ must be positive, at least for convex domains $D$. We have also the estimates $\|z\|_{1} \leq \mathscr{C}^{\prime}\|H\|_{0}$ and $\|H\|_{0} \leq|\xi| \mathscr{C}^{\prime \prime}\|z\|_{1}$, with constants $\mathscr{C}^{\prime}$ and $\mathscr{C}^{\prime \prime}$ depending on the domain $D$ and on the coefficient $c$. The estimate $|\xi| \geq 1 / \mathscr{C}^{\prime} \mathscr{C}^{\prime \prime}$ is a consequence.

If $D$ is a disk, a computation shows that the lowest eigenvalue $\xi_{1}$ has the value

$$
\xi_{1}=\frac{i c}{2} \frac{J_{0}(i c)}{J_{1}(i c)}=\frac{1+c^{2} / 4+\cdots}{1+c^{2} / 8+\cdots} .
$$

We see that $\xi_{1}>1$ for $c>0$ and $\xi_{1}=1$ for $c=0$. It follows that, for a disk $D$, there is one situation-admissible on the basis of our structural condition (3)in which there is nonuniqueness for the linear boundary value problem (49)-(52), namely, the case $\alpha=H_{0}=0$ and $\beta=\gamma$ or $\gamma / \beta=\xi_{1}$. This case leads to the integrand $\Phi=\beta\left(H^{2}-K\right)$. Here

$$
z=\frac{a}{2}\left(x^{2}+y^{2}-1\right), \quad H=a
$$

with an arbitrary constant $a$, is a continuum of solutions for (53), (54), (55), (56) for $\xi=1$. This fact has already been mentioned in the introduction.

For an annulus $D=\left\{(x, y) ; r^{2}<x^{2}+y^{2}<1\right\}$, that is, for an interface with a circular hole, a computation for the case $c=0$ shows for instance that no eigenvalue $\xi$ of (53)-(56) can lie in the interval $0 \leq \xi \leq 1$.

13. We now assume that the quotient $\gamma / \beta$ is different from the eigenvalues of (53)-(56). Then the solutions $z^{(0)}(x, y)$ and $H^{(0)}(x, y)$ of the linear problem (49)(52) are unique and, by the theory of partial differential equations,

$$
\|z, H\| \leq \mathscr{C}_{1}\left(\|\varphi\|_{4, \lambda}^{\partial D}+\left|H_{0}\right|\right) .
$$

Here $\mathscr{C}_{1}$ is a bound depending only on the domain $D$, on the material parameters $\alpha, \beta, \gamma$ and on a bound for $H_{0}$ (reflecting the presence of $H_{0}$ in the coefficient on the left-hand side of (51) and the abbreviated notation

$$
\|z, H\|=\|z\|_{4, \lambda}^{\bar{D}}+\|H\|_{2, \lambda}^{\bar{D}}
$$

is used.

The solution $z(x, y), H(x, y)$ of the full system (42), (43), (44), (45) is now sought in the form

$$
z(x, y)=z^{(0)}(x, y)+z^{(1)}(x, y), \quad H(x, y)=H^{(0)}(x, y)+H^{(1)}(x, y) .
$$


The functions $z^{(1)}$ and $H^{(1)}$ satisfy the conditions

$$
\begin{aligned}
\Delta z^{(1)}-2 H^{(1)} & =f(z, H), & & (x, y) \in D, \\
\Delta H^{(1)}-2\left(\frac{\alpha}{\beta}+H_{0}^{2}\right) H^{(1)} & =g(z, H), & & (x, y) \in D, \\
z^{(1)} & =0, & & (x, y) \in \partial D, \\
\beta H^{(1)}-\gamma \kappa(\sigma) \frac{\partial z^{(1)}}{\partial \nu} & =h(\varphi, z), & & (x, y) \in \partial D .
\end{aligned}
$$

Assume for the moment that the right-hand sides $f, g$, and $h$ in (63), (64), (66) are functions of $(x, y)$ and of $\sigma$, respectively. Then the following estimate is true:

$$
\left\|z^{(1)}, H^{(1)}\right\| \leq \mathscr{C}_{2}\left(\|f\|_{2, \lambda}^{\bar{D}}+\|g\|_{0, \lambda}^{\bar{D}}+\|h\|_{2, \lambda}^{\partial D}\right) .
$$

As a consequence of $(60)$, we have of course

$$
\|z, H\| \leq\left\|z^{(1)}, H^{(1)}\right\|+\mathscr{C}_{1}\left(\|\varphi\|_{4, \lambda}^{\partial D}+\left|H_{0}\right|\right) .
$$

Given the nonlinear structure of the expressions $f, g, h$, the existence for the solutions of the problem (63)-(66) can be proved with the help of an iteration scheme, provided $\|\varphi\|_{4, \lambda}^{\partial D}<m$ and $\left|H_{0}\right|<m$, and the positive bound $m$ is chosen sufficiently small.

This completes the proof of Theorem 1A. For related proofs and details see, e.g., [54, pp. 360-364], [55, pp. 21-26].

As for the volume constraint problem, the following theorem holds.

THeOREM 1B. There is a positive constant $m$ depending only on the domain $D$, on the material parameters $\alpha, \beta, \gamma$ and on a bound for $H_{0}$ with the following property. If $\|\varphi\|_{4, \lambda}^{\partial D}<m,\left|H_{0}\right|<m$, and $|\mu|<m$, then the problem (14), (30), (38), (39) has a unique solution $z(x, y)$ in the regularity class $C^{4, \lambda}(\bar{D})$ satisfying an inequality

$$
\|z\|_{4, \lambda}^{\bar{D}} \leq \mathscr{C}\left\{\|\varphi\|_{4, \lambda}^{\partial D}+\left|H_{0}\right|+|\mu|\right\} .
$$

Here the bound $\mathscr{C}$ is independent of $\varphi$.

14. To interpret the boundary conditions associated with Problem 2, let us consider the situation described in Sec. 7 and assume that the extremal surface $\mathscr{S}$ spans the interior of a region $\mathscr{B}$ in $R^{3}$ so that the boundary $\partial \mathscr{S}$ is required to lie on the boundary $\partial \mathscr{B}$ of this region. In this situation, the variation vector $\mathbf{y}$ must be parallel to $\partial \mathscr{B}$, but is otherwise arbitrary. An inspection of (7)-(9), (27) then shows that, on the boundary $\partial \mathscr{S}$,

$$
\left(\mathbf{y}, \alpha \mathbf{X}+\beta\left(H-H_{0}\right)^{2} \mathbf{X}-\gamma K \mathbf{X}+\mu \mathbf{Q}(\mathbf{x}), d \mathbf{x}\right)=0
$$

for all such $\mathbf{y}$. Given that $d \mathbf{x}$ is also parallel to $\partial \mathscr{B}$ and that the same is true for $\mathbf{Q}(\mathbf{x})$, it follows that the vector $\left\{\alpha+\beta\left(H-H_{0}\right)^{2}-\gamma K \mathbf{X}\right\} \mathbf{X}+\mu \mathbf{Q}(\mathbf{x})$ is tangent to $\partial \mathscr{B}$ in all boundary points of $\mathscr{S}$. In other words: A potential solution surface must meet the boundary of the region $\mathscr{B}$ at a right angle. 
If there is no volume constraint, the term $\mu \mathbf{Q}(\mathbf{x})$ is missing in (70), but this fact does not alter the conclusion (74).

In the presence of a wetting energy term, (70) leads to the condition

$$
\alpha \mathbf{X}+\beta\left(H-H_{0}\right)^{2} \mathbf{X}-\gamma K \mathbf{X}+\mu \mathbf{Q}(\mathbf{x})=\nu \mathbf{N}(\mathbf{x}) .
$$

Scalar multiplication with the vector $\mathbf{N}(\mathbf{x})$ results in the boundary condition

$$
\left\{\alpha+\beta\left(H-H_{0}\right)^{2}-\gamma K \mathbf{X}\right\} \cos \chi=\nu .
$$

Here $\chi$ denotes the angle at which the surface $\mathscr{S}$ meets the boundary $\partial \mathscr{B}$ (the angle between $\mathbf{X}$ and $\mathbf{N})$. Note that for the special case $\beta=0$, i.e., $\mathscr{E}(\mathscr{S}) \equiv \iint_{\mathscr{S}} d A$, (72) is reduced to the condition of constant contact angle $\chi$ which plays an important role in the theory of capillarity. For the time being, we shall neglect the wetting energy term.

Regarding the boundary condition corresponding to the other conditions (22) and (23), note the following. Now that we know that all solution surfaces $\mathscr{S}$ must meet the boundary of $\mathscr{B}$ at a right angle, we can restrict our further discussion to a consideration of variation vectors $\mathbf{y}$ which are parallel to the normal vector $\mathbf{X}$ on the boundary of $\mathscr{S}$. The quantity $\mathbf{y} \cdot \mathbf{X}$ is arbitrary, but the normal derivative $\partial(\mathbf{y} \cdot \mathbf{X}) / \partial n$ must vanish at $\partial \mathscr{S}$. Therefore, an application of (8) and (9) leads to the boundary condition

$$
\beta \frac{\partial H}{\partial n}+\gamma \Lambda^{(2)}=0
$$

which replaces $(32)$.

15. To illustrate Problem 2 for surfaces in nonparametric representation $z=$ $z(x, y)$, let $D$ be a domain in the $(x, y)$-plane, as in Sec. 11 before, and consider the cylinder $\mathscr{Z}$ erected over the boundary $\partial D$ with generating lines parallel to the $z$-axis. The boundary of an admissible surface $\mathscr{S}$ is a curve which must lie on $\mathscr{Z}$, but is now not otherwise restricted; it will have a representation $x=x(\sigma), y=y(\sigma)$, $z=z(x(\sigma), y(\sigma)) \equiv \varphi(\sigma)$. We shall denote this curve again by $\Gamma$; it is not fixed now.

As we have seen, a potential solution surface must meet the bounding cylinder at a right angle. This is equivalent to the boundary condition

$$
\frac{\partial z}{\partial \nu}=0, \quad(x, y) \in \partial D .
$$

(As in Sec. 11, $\partial / \partial \nu$ denotes differentiation with respect to the outer normal of $D$.) As for the boundary condition corresponding to (73), it is necessary to work out the form of $\Lambda^{(2)}$ in terms of the function $z(x, y)$ and the geometric properties of the domain $D$.

Using the notations of Sec. 4, a computation gives, in addition to (37), now with 
the relation $\partial z / \partial \nu=0$ incorporated,

$$
\begin{gathered}
k=\frac{1}{\sqrt{1+\varphi^{\prime 2}}} \sqrt{\varphi^{\prime \prime 2}+\kappa^{2}\left(1+\varphi^{2}\right)}, \\
\tau=\frac{\kappa \varphi^{\prime \prime \prime}-\kappa^{\prime} \varphi^{\prime \prime} \kappa^{3} \varphi^{\prime}}{\varphi^{\prime \prime 2}+\kappa^{2}\left(1+\varphi^{\prime 2}\right)}, \\
\cos \vartheta=\frac{\varphi^{\prime \prime}}{\sqrt{\varphi^{\prime \prime 2}+\kappa^{2}\left(1+\varphi^{\prime 2}\right)}} .
\end{gathered}
$$

It follows from these relations that $(18)$ is

$$
\Lambda^{(2)}=\frac{\partial}{\partial s} \frac{\kappa \varphi^{\prime}}{1+\varphi^{\prime 2}},
$$

and the boundary condition (22) becomes

$$
\beta \frac{\partial H}{\partial \nu}+\gamma \frac{\partial}{\partial \sigma} \frac{\kappa \partial z / \partial \sigma}{1+(\partial z / \partial \sigma)^{2}}=0 .
$$

Recall that $d s^{2}=\left(1+\varphi^{\prime 2}\right) d \sigma^{2}$. As a consequence of (79), we have

$$
\iint_{D} \Delta H d x d y=\int_{\partial D} \frac{\partial H}{\partial \nu} d \sigma=0 .
$$

In view of (74), an integration of (14) gives also

$$
\iint_{D} H d x d y=\frac{1}{2} \int_{\partial D} \frac{1}{W} \frac{\partial z}{\partial \nu} d \sigma=0 .
$$

Both relations (80) and (81) are obviously necessary conditions for the solvability of the nonparametric free boundary value problem.

In its complete form, Problem 2 comprises the following: The differential equations (14) and (21) (for Problem 2a) or (14) and (30) (for Problem 2b), to be satisfied in the domain $D$, and the boundary conditions (74) and (79), to be satisfied on the boundary $\partial D$. For both differential equations, these are Neumann conditions. Note that the quantity $H_{0}$ is not contained in the boundary conditions. This quantity appears only in the (negative) coefficient of $H$ on the left-hand side of (43) and as a factor of the quadratic term $\left(z_{x x} z_{y y}-z_{x y}^{2}\right) / W^{4}$ in the function $h$ on the right-hand side of (43).

The differential equations are thus (42) and (43); they are complemented by the boundary conditions (74) and

$$
\beta \frac{\partial H}{\partial \nu}+\gamma \frac{\partial}{\partial \sigma}\left(\kappa(\sigma) \frac{\partial z}{\partial \sigma}\right)=\bar{h}\left(\frac{\partial z}{\partial \sigma}, \frac{\partial^{2} z}{\partial \sigma^{2}}\right),
$$

where

$$
\bar{h}\left(\frac{\partial z}{\partial \sigma}, \frac{\partial^{2} z}{\partial \sigma^{2}}\right)=-\frac{\partial}{\partial \sigma}\left(\frac{\kappa(\sigma)(\partial z / \partial \sigma)^{3}}{1+(\partial z / \partial \sigma)^{2}}\right) .
$$


For the present situation, the linearized boundary value problem corresponding to (49) $-(52)$ is

$$
\begin{aligned}
\Delta z-2 H & =0, & & (x, y) \in D, \\
\Delta H-c^{2} H & =0, & & (x, y) \in D, \\
\frac{\partial z}{\partial \nu} & =0, & & (x, y) \in \partial D, \\
\frac{\partial H}{\partial \nu}+\xi \frac{\partial}{\partial \sigma}\left(\kappa(\sigma) \frac{\partial z}{\partial \sigma}\right) & =0, & & (x, y) \in \partial D .
\end{aligned}
$$

Here we have again set $c^{2}=2\left(\alpha+\beta H_{0}^{2}\right) / \beta$ and $\xi=\gamma / \beta$.

Integrating the difference $H \Delta z-z \Delta H$, we find

$$
\iint_{D}\left\{4 H^{2}+c^{2}\left(z_{x}^{2}+z_{y}^{2}\right)\right\} d x d y+2 \xi \int_{\partial D} \kappa(\sigma)\left(\frac{\partial z}{\partial \sigma}\right)^{2} d \sigma=0 .
$$

It follows from this that the problem $(84)-(87)$ has only the trivial solution $z=$ const, $H=0$, at least for a convex domain $D$.

Note that neither the differential equations nor the boundary conditions contain the function $z(x, y)$ explicitly, so that $z(x, y)+$ const is a solution of the boundary value problem if $z(x, y)$ is. We can therefore stipulate that any solution surface should satisfy the condition $z\left(x_{0}, y_{0}\right)=0$ for an interior point $\left(x_{0}, y_{0}\right)$ of $D$.

As a consequence of the foregoing, we have

Theorem 2A. Assume that the domain $D$ is convex or that the problem (84)-(87) has no nontrivial solutions. There is a positive constant $m$ depending only on $D$, on the material constants $\alpha, \beta, \gamma$ and on a bound for the spontaneous curvature $H_{0}$ with the following property. The only solution $z(x, y)$ of Problem 2(a) of regularity class $C^{4, \lambda}(\bar{D})$ satisfying the inequality

$$
\|z\|_{4, \lambda}^{\bar{D}} \leq m
$$

is $z(x, y) \equiv 0$.

It is remarkable and somewhat surprising on physical grounds that Theorem $2 \mathrm{~A}$ is valid regardless of the value of $H_{0}$. Of course, the spontaneous curvature enters the energy expression which, for solution surfaces with sufficiently small norm $\left(\|z\|_{4, \lambda}^{\bar{D}} \leq m\right)$, is

$$
\mathscr{E}(\mathscr{S})=\left(\alpha+H_{0}^{2}\right)|D|+O(m) .
$$

Here $|D|$ denotes the area of the domain $D$. At the present stage, the situation for nonconvex domains is not decided. It would be of interest to study the solvability of the homogeneous system (84)-(87), as well as the possibility of solution surfaces of Problem 2(a) for general domains.

Regarding the volume constraint free boundary value problem, the differential equation (21) or (43) has to be replaced by the differential equation (30) or

$$
\Delta H-2\left(\frac{\alpha}{\beta}+H_{0}^{2}\right) H=\frac{\mu}{\beta}+g(z, H),
$$


and equation $(85)$ becomes

$$
\Delta H-c^{2} H=\frac{\mu}{\beta} .
$$

The Lagrange multiplier $\mu$ assumes the role of a control parameter, leading to a one-parameter family of solution surfaces.

Theorem 2B. Assume that the domain $D$ is convex or that the problem (84)-(87) has no nontrivial solutions. There is a positive constant $m$ depending only on $D$, on the material constants $\alpha, \beta, \gamma$ and on a bound for the spontaneous curvature $H_{0}$ with the following property. If $|\mu|<m$, then Problem 2(b) has a unique solution $z(x, y)$ in the regularity class $C^{4, \lambda}(\bar{D})$ satisfying an inequality

$$
\|z\|_{4, \lambda}^{\bar{D}} \leq \mathscr{C}|\mu| .
$$

Here the bound $\mathscr{C}$ is independent of $\mu$.

The proof is similar to those of Theorems 1A, 1B and follows again the lines of the proof given in $[55$, p. 21-26].

16. The Theorems 1A, 1B, 2A, 2B of the preceding sections are local in nature. Further extremal surfaces may well exist; obviously, if they do, their norms must be sufficiently large. To see this clearly, let us consider the simplest one-dimensional case of Problem 2(a), namely a stationary solution surface $\mathscr{S}$ bounded by two vertical walls, say, the planes $x=0$ and $x=x_{1}>0$. The plane portion with the position vector $(x, y, 0)$ for $0 \leq x \leq x_{1},-\infty<y<\infty$ is always a solution. The free energy associated with it is the smallest possible. But there will be other solution surfaces. For these, the position vector $\mathbf{x}$ has the form $(x(s), t, z(s))$, where the parameter $s$ may be taken as the arc length of the profile curve $x=x(s), z=z(s)$, so that $\mathscr{S}$ is defined over an infinite strip $0 \leq s \leq s_{1},-\infty<t<\infty$ of the $(s, t)$-plane. Also the mean curvature of $\mathscr{S}$ will be a function $H(s)$ of $s$ alone, while the Gaussian curvature of $\mathscr{S}$ is zero, given that $\mathscr{S}$ is a ruled surface. We still define the functions $p(s)=d x / d s$ and $q(s)=d z / d s$ so that $p^{2}(s)+q^{2}(s)=1$. The differential equations (15) and (21) can now be reduced to the simple system

$$
\begin{aligned}
p^{\prime}(s) & =-2 H(s) q(s), \\
q^{\prime}(s) & =2 H(s) p(s), \\
H^{\prime \prime}(s) & =-2 H^{3}(s)+c^{2} H(s) .
\end{aligned}
$$

Here, as before, $c^{2}=2\left(\alpha+\beta H_{0}^{2}\right) / \beta$. The boundary conditions become

$$
\begin{gathered}
x(0)=0, \quad x\left(s_{1}\right)=x_{1} ; \quad p(0)=p\left(s_{1}\right)=1 ; \\
q(0)=q\left(s_{1}\right)=0 ; \quad H^{\prime}(0)=H^{\prime}\left(s_{1}\right)=0 .
\end{gathered}
$$

Moreover, we shall assume that $H(0)=-a$, where $a$ is a positive number still to be chosen. (This is no restriction since equation (96) is invariant under the change $H \rightarrow-H$.) For small positive values of $s$, the mean curvature has an expansion

$$
H(s)=-a+a\left(a^{2}-\frac{c^{2}}{2}\right) s^{2}-\frac{a}{2}\left(a^{2}-\frac{c^{2}}{2}\right)\left(a^{2}-\frac{c^{2}}{6}\right) s^{4}+\cdots .
$$


A first integral of $(96)$ is

$$
H^{\prime 2}(s)=\left(a^{2}-H^{2}(s)\right)\left\{a^{2}-c^{2}+H^{2}(s)\right\} .
$$

Once the function $H(s)$ has been found, its values can be substituted in the equations (94), (95) for a determination of the functions $p(u)$ and $q(u)$.

The nature of the solutions $H(u)$ of $(96)$ depends on the size of the initial value $a$. For $a=c / \sqrt{2}$, the mean curvature will have the constant value $H=c / \sqrt{2}$, and $\mathscr{S}$ is a circular cylinder (not a solution for our present problem).

Assume that $a>c$. In this case, we introduce a new parameter $u$ with the help of the substitution $H=-a \cos u$ so that

$$
s=s(u)=\int_{0}^{u} \frac{d u}{\sqrt{a^{2}-c^{2}+a^{2} \cos ^{2} u}} .
$$

The differential equations for $p$ and $q$ become

$$
\begin{aligned}
& \frac{d p}{d u}=\frac{2 a \cos u}{\sqrt{a^{2}-c^{2}+a^{2} \cos ^{2} u}} q, \\
& \frac{d q}{d u}=-\frac{2 a \cos u}{\sqrt{a^{2}-c^{2}+a^{2} \cos ^{2} u}} p .
\end{aligned}
$$

Now observe that

$$
I(u) \equiv \int_{0}^{u} \frac{a \cos u}{\sqrt{a^{2}-c^{2}+a^{2} \cos ^{2} u}} d u=\sin ^{-1}\left(\frac{a \sin u}{\sqrt{2 a^{2}-c^{2}}}\right) .
$$

From this it follows that the solutions of (101), (102) are

$$
p=\cos 2 I(u)=1-2 \sin ^{2} I(u)=\frac{2 a^{2} \cos ^{2} u-c^{2}}{2 a^{2}-c^{2}}
$$

and

$$
q=\sin 2 I(u)=2 \sin I(u) \cos I(u)=\frac{2 a \sin u \sqrt{a^{2}-c^{2}+a^{2} \cos ^{2} u}}{2 a^{2}-c^{2}} .
$$

Thus, ultimately we find the following representation for the profile curve of $\mathscr{S}$ :

$$
\begin{gathered}
x(u)=\frac{1}{2 a^{2}-c^{2}} \int_{0}^{u} \frac{2 a^{2} \cos ^{2} w-c^{2}}{\sqrt{a^{2}-c^{2}+a^{2} \cos ^{2} w}} d w, \\
z(u)=\frac{2 a}{2 a^{2}-c^{2}} \cos u, \\
H(u)=-a \cos u .
\end{gathered}
$$

We see that the boundary conditions (97) are satisfied if we let the parameter $u$ range over the interval $0 \leq u \leq \pi$. Then

$$
x_{1}=\frac{1}{2 a^{2}-c^{2}} \int_{0}^{\pi} \frac{2 a^{2} \cos ^{2} u-c^{2}}{\sqrt{a^{2}-c^{2}+a^{2} \cos ^{2} u}} d u .
$$

For a given value of the constant $c$, it is clear that $x_{1} \rightarrow 0$ for $a \rightarrow c+$ and $x_{1} \rightarrow \infty$ for $a \rightarrow \infty$. 


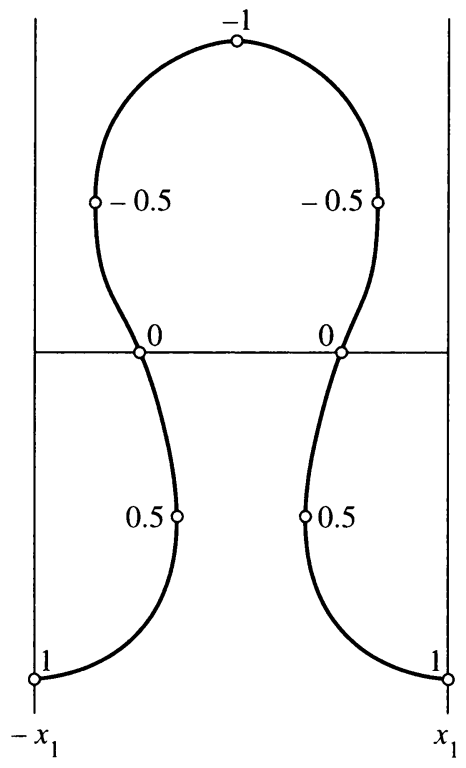

FIG. 1. Profiles of two distinct solution surfaces for Problem 2(a) (Case $c^{2}=1 / 2$, markings indicate values of the mean curvature)

The corresponding surface $\mathscr{S}$ meets the bounding planes $x=0$ and $x=x_{1} \equiv$ $x(\pi)$ at right angles, and $\partial H / \partial n=0$ at these planes. Thus, $\mathscr{S}$ is a solution of Problem 2(a) for our bounding configuration. In view of the reflection principle of $[55$, p. 19], $\mathscr{S}$ can also be analytically extended by reflection on the vertical planes. Fig. 1 shows the profile of the extended surface $\mathscr{S}$ between the planes $-x_{1}$ and $x_{1}$ for the case $c^{2}=1 / 2, a=1$. Here $x_{1}=0.80574$.

A repetition of the reflection process leads to a periodic undulating profile, and any portion between two extrema is a possible solution of Problem 2(a).

It is clear from the symmetries of $\mathscr{S}$ shown in Fig. 1 that $\mathscr{S}$ is also a solution surface of Problem 2(b), for the value $\mu=0$ of the Lagrange multiplier in the differential equation (30). Note that

$$
H^{\prime 2}(s)=\left(a^{4}-H^{4}(s)\right)-c^{2}\left(a^{2}-H^{2}(s)\right)+\frac{2 \mu}{\beta}(a-H(s))
$$

is a first integral of the differential equation (96). Again, in addition to the solution surface of Problem 2(b) with small norm stipulated in Theorem 2B, there may be further extremal surfaces.

Equations (94), (95), (96) are equivalent to the differential equation for elastica investigated by L. Euler (Opera omnia (1) 24, pp. 233-234, 263), and Fig. 1 is an example of his fourth class (i.e., Fig. 7 on p. 248).

17. The foregoing discussion provides the outline for a theory of boundary value problems connected with the energy functional $\mathscr{E}(\mathscr{S})$ of $(1),(2)$. This theory leads 
to a variety of questions for which answers have yet to be found. The problems of interest include:

(1) Solvability of specific boundary value problems for auxiliary linearized equations, e.g., (53)-(56), (84)-(87) in general domains, with or without corners;

(2) Compactness properties for minimizing sequences of surfaces;

(3) Existence of global energy minimizing, or energy stationary, solution surfaces;

(4) Analogies with Douglas's sufficient condition and Courant's condition of cohesion for the case of several boundaries;

(5) Regularity-interior and on the boundary-of these surfaces;

(6) Local behavior near a corner of the boundary;

(7) Stability and energy estimates;

(8) Number of solution surfaces;

(9) Isolatedness and rigidity (in the sense of [50; 53, pp. 7-9]) of the solution surfaces;

(10) Geometric properties of the solution surfaces;

(11) Existence of unsymmetrical solutions for the case of symmetrical boundaries;

(12) Information about the topological type of embedded solution surfaces.

The situation is quite complex. This is of course not surprising if one considers that many of the above questions have not yet found definitive answers even for the boundary value problems associated with minimal surfaces and (in the presence of a volume constraint) with surfaces of constant mean curvature. It is for physical reasons that we are looking for embedded extremal surfaces, even though the existence proofs often produce immersed surfaces, that is, differential geometric regular surfaces with possible self-intersections. For example, a knotted contour cannot bound an embedded surface of the type of the disk.

Among the basic theorems of particular interest is the following:

THEOREM. Let $\Gamma$ be an unknotted rectifiable Jordan curve of finite total curvature. There exists an embedded disk-type surface $\mathscr{S}=\{\mathbf{x}=\mathbf{x}(u, v) ;(u, v) \in \bar{P}\}, P$ the unit disc, bounded by $\Gamma$ (in the customary sense; see, e.g., [54, Sec. 292] of minimal energy $\mathscr{E}$. The position vector $\mathbf{x}(u, v)$ is analytic for $(u, v) \in P$ and continuous for $(u, v) \in \bar{P}$. If $\Gamma$ possesses higher regularity, say $\Gamma \in C^{m, \lambda}$, where $m \geq 4$, $0<\lambda<1$, then $\mathbf{x}(u, v)$ belongs to the regularity class $C^{m, \lambda}(\bar{P})$.

\section{REFERENCES}

[1] D. M. Anderson, Studies in the microstructure of microemulsion, Dissertation, Univ. of Minnesota, 1986

[2] D. M. Anderson, H. T. Davis, J. C. C. Nitsche, and L. E. Scriven, Periodic surfaces of prescribed mean curvature, Advances in Chem. Phys., vol. 77, Interscience, New York, 1990, pp. 337-396

[3] D. M. Anderson, S. M. Gruner, and S. Leibler, Geometric aspects of the frustration in the cubic phases of lyotropic liquid crystals, Proc. Nat. Acad. Sci. U.S.A. 85, 5364-5368 (1988)

[4] S. Balasundaram and P. K. Bhattacharyya, On existence of solution of the Dirichlet problem of fourth order partial differential equations with variable coefficients, Quart. Appl. Math. 41, 311317 (1983)

[5] S. N. Bernstein, Sur les surfaces définies au moyen de leur courhure moyenne et totale, Ann. Sci. École Norm. Sup. 27, 233-256 (1910)

[6] R. Bryant, A duality theorem for Willmore surfaces, J. Differential Geom. 20, 23-53 (1984) 
[7] F. Casorati, Mesure de la courbure des surfaces suivant l'idée commune. Ses rapports avec les mesures de courbure Gaussienne et moyenne, Acta Math. 14, 95-110 (1890/91)

[8] B. Y. Chen, An invariant of conformal mappings, Proc. Amer. Math. Soc. 40, 563-564 (1973)

[9] B. Y. Chen, Some conformal invariants of submanifolds and their applications, Boll. Un. Mat. Ital. (4) 10, 380-385 (1974)

[10] B. Y. Chen, On a variational problem on hypersurfaces, J. London Math. Soc. (2) 6, 321-325 (1973)

[11] H. T. Davis and L. E. Scriven, Stress and structure in fluid interfaces, Advances in Chem. Phys. 49, 357-454 (1982)

[12] G. Dziuk, Über quasilineare Systeme mit isothermen Parametern an Ecken der Randkurven, Analysis 1, 63-81 (1981)

[13] G. Dziuk, Über die Stetigkeit teilweise freier Minimalflächen, Manuscripta Math. 36, 241-251 (1981)

[14] W. Fenchel, Über Krümmung und Windung geschlossener Raumkurven, Math. Ann. 101, 238-252 (1929)

[15] R. Finn, Remarks relevant to minimal surfaces and to surfaces of prescribed mean curvature, J. Analyse Math. 14, 139-160 (1965)

[16] W. Fischer and E. Koch, New surface patches for minimal balance surfaces. I, Branched catenoids, Acta Cryst. A 45, 166-169 (1989); II, Multiple catenoids, Acta Cryst. A 45, 169-174 (1989); III, Infinite strips, Acta Cryst. A 45, 485-490 (1989); IV, Catenoids with spout-like attachments, Acta Cryst. A 45, 558-563 (1989)

[17] A. Fogden, S. T. Hyde, and G. Lundberg, Bending energy of surfactant films, J. Chem. Soc. Faraday Trans. 87 (7), 949-955 (1991)

[18] P. Funk, Variationsrechnung und ihre Anwendung in Physik und Technik, 2nd ed., Springer-Verlag, Berlin, Heidelberg, and New York, 1970

[19] C. Gerhardt, Existence, regularity, and boundary behaviour of generalized surfaces of prescribed mean curvature, Math. Z. 139, 173-198 (1974)

[20] G. Gerhardt, Boundary value problems for surfaces of prescribed mean curvature, J. Math. Pures Appl. 58, 75-109 (1979)

[21] S. Germain, Recherches sur la théorie des surfaces élastiques, Imprimerie de Huzard-Courcier, Paris, 1921

[22] M. Giaquinta, Regolarità delle superfici $B V(\Omega)$ con curvatura media assegnata, Boll. Un. Mat. Ital. 8, 567-578 (1973)

[23] M. Giaquinta, On the Dirichlet problem for surfaces of prescribed mean curvature, Manuscripta Math. 12, 73-86 (1974)

[24] D. Gilbarg and N. S. Trudinger, Elliptic partial differential equations of second order, 2nd ed., Springer-Verlag, Berlin, Heidelberg, New York, and Tokyo, 1983

[25] E. Giusti, Boundary value problems for non-parametric surfaces of prescribed mean curvature, Ann. Scuola Norm. Sup. Pisa (4) 3, 501-548 (1976)

[26] E. Giusti, On the equation of surfaces of prescribed mean curvature, Invent. Math. 46, 111-137 (1978)

[27] P. Grisvard, Elliptic problems in nonsmooth domains, Monographs and Studies in Math., vol. 24, Pitman Advanced Pub. Program, Boston, 1985

[28] S. M. Gruner, Stability of lyotropic phases with curved interfaces, J. Phys. Chem. 93, 7562-7570 (1989)

[29] M. Grüter, S. Hildebrandt, and J. C. C. Nitsche, On the boundary behavior of minimal surfaces with a free boundary which are not minima of the area, Manuscripta Math. 35, 387-410 (1981)

[30] M. Grüter, S. Hildebrandt, and J. C. C. Nitsche, Regularity for surfaces of constant mean curvature with free boundaries, Acta Math. 156, 119-152 (1986)

[31] E. Heinz, Über Flächen mit eineindeutiger Projektion auf eine Ebene, deren Krümmungen durch Ungleichungen eingeschränkt sind, Math. Ann. 129, 451-454 (1955)

[32] E. Heinz, On the nonexistence of a surface of constant mean curvature with finite area and prescribed rectifiable boundary, Arch. Rational Mech. Anal. 35, 249-252 (1969)

[33] E. Heinz, Minimalflächen mit polygonalem Rand, Math. Z. 183, 547-564 (1983)

[34] W. Helfrich, Elastic properties of lipid bilayers: Theory and possible experiments, Z. Naturforsch. A 28c, 693-703 (1973)

[35] W. Helfrich and H. Rennschuh, Landau theory of the lamellar-to-cubic phase transition, Colloque de Physique C7, 51, supp. au no. 23, 189-195 (1990) 
[36] S. Hildebrandt and J. C. C. Nitsche, A uniqueness theorem for surfaces of least area with partially free boundaries on obstacles, Arch. Rational Mech. Anal. 79, 189-218 (1982)

[37] S. T. Hyde, Curvature and the global structure of interfaces in surfactant-water systems, Colloque de Physique C7, 51, suppl. au no. 23, 207-228 (1990)

[38] S. T. Hyde, I. S. Barnes, and B. W. Ninham, Curvature energy of surfactant interfaces confined to the plaquettes of a cubic lattice, Langmuir, ACS J. Surf. Colloids 6, 1055-1062 (1990)

[39] J. N. Israelachvili, Intermolecular and surface forces: With applications to colloidal and biological systems, Academic Press, London and Orlando, 1985

[40] J. N. Israelachvili and P. M. McGuiggan, Forces between surfaces in liquids, Science 241, 795-800 (1988)

[41] N. Kapouleas, Complete constant mean curvature surfaces in euclidean three-space, Ann. of Math. 131, 239-330 (1990)

[42] H. Karcher, The triply periodic minimal surfaces of Alan Schoen and their constant mean curvature companions, Manuscripta Math 64, 291-337 (1989)

[43] H. Karcher, U. Pinkall, and I. Sterling, New minimal surfaces in $S^{3}$, J. Differential Geom. 28, 169-185 (1988)

[44] G. R. Kirchhoff, Über das Gleichgewicht und die Bewegung einer elastischen Scheibe, J. Reine Angew. Math. 40, 51-88 (1850)

[45] K. L. Kirk, S. M. Gruner, and D. L. Stein, Thermodynamic model of the lamellar to inverse hexagonal phase transition of lipid membrane-water systems, Biochem. 23, 1093-1102 (1984)

[46] V. A. Kondrat' ev and O. A. Oleinik, Boundary value problems for partial differential equations in non-smooth domains, Russian Math. Surv. 38, 1-86 (1983)

[47] H. B. Lawson, Complete minimal surfaces in $S^{3}$, Ann. of Math. (2) 92, 335-374 (1970)

[48] A. E. H. Love, A treatise on the mathematical theory of elasticity, 2nd ed., Cambridge University Press, Cambridge, 1906

[49] H. Minkowski, Kapillarität, Encyclopedia Math. Wissen. 5.1.9, B. G. Teubner, Leipzig, 1903-1921 (completed 1906), pp. 558-613

[50] J. C. C. Nitsche, Concerning the isolated character of solutions of Plateau's problem, Math. Z. 109, 393-411 (1969)

[51] J. C. C. Nitsche, Vorlesungen über Minimalflächen, Springer-Verlag, Berlin, Heidelberg, and New York, 1975

[52] J. C. C. Nitsche, A volume formula, Analysis 3, 337-346 (1983)

[53] J. C. C. Nitsche, Stationary partitioning of convex bodies, Arch. Rational Mech. Anal. 89, 1-19 (1985); corrigendum in Arch. Rational Mech. Anal. 95, 389 (1986)

[54] J. C. C. Nitsche, Lectures on minimal surfaces, Cambridge University Press, Cambridge, New York, New Rochelle, Melbourne, and Sydney, 1989

[55] J. C. C. Nitsche, Periodic surfaces which are extremal for energy functionals containing curvature functions, IMA Preprint no. 785, March 1991 (to appear in Proc. Workshop Statistical Thermodynamics and Differential Geometry of Microstructured Materials, H. T. Davis and J. C. C. Nitsche, eds., IMA vol. in Math. and its Appl.)

[56] J. C. C. Nitsche, Mathematik in Berlin, Born konkreter Geometrie über die Jahrhunderte, Wissenschaft und Stadt (D. Heckelmann and O. Büsche, eds.), Colloquium Verlag, Berlin (to appear)

[57] Ou-Yang Zhong-can, Anchor ring vesicle membranes, Phys. Rev. A 41, 4517-4520 (1990)

[58] Ou-Yang Zhong-can and W. Helfrich, Bending energy of vesicle membranes: General expressions for the first, second, and third variation of the shape energy and application to spheres and cylinders, Phys. Rev. A 39, 5280-5288 (1989)

[59] S. D. Poisson, Mémoire sur les surfaces élastiques, Cl. Sci. Mathém. Phys. Inst. de France, 2nd pt., 167-225 (1812)

[60] S. D. Poisson, Mémoire sur le calcul des variations, Mém. Acad. Roy. Sci. Inst. de France 12, 223-331 (1833)

[61] E. Reissner, The effect of transverse shear deformation on the hending of the elastic plates, J. Appl. Mech. 12, A68-A77 (1945)

[62] E. Reissner, On bending of elastic plates, Quart. Appl. Math. 5, 55-68 (1947)

[63] J. S. Rowlinson and B. Widom, Molecular theory of capillarity, Clarendon Press, Oxford, 1982

[64] M. Schäfer, Über eine Verfeinerung der klassischen Theorie dünner schwach gebogener Platten, Z. Angew. Math. Mech. 32, 161-171 (1952) 
[65] A. H. Schoen, Infinite periodic minimal surfaces without self-intersections, NASA Techn. Rep. D-5541 (1970)

[66] H. A. Schwarz, Gesammelte Mathematische Abhandlungen, vol. 1, Springer, Berlin, 1890

[67] H. A. Schwarz, Zur Theorie der Minimalflächen, deren Begrenzung aus geradlinigen Strecken besteht, Sitz.-Ber. Königl. Preuss., Akad. d. Wiss. Berlin, Phys.-Math. Cl., 1237-1266 (1894)

[68] L. E. Scriven, Equilibrium bicontinuous structure, Nature 263, 123-125 (1976)

[69] J. Serrin, The problem of Dirichlet for quasilinear elliptic differential equations with many independent variables, Philos. Trans. Roy. Soc. London Ser. A 264, 413-496 (1969)

[70] L. Simon, Existence of Willmore surfaces, Proc. Centre Math. Anal., Austra. Nat. Univ. 10, 187$216(1985)$

[71] I. Sterling, Willmore surfaces and computers, (to appear in Proc. Workshop Statistical Thermodynamics and Differential Geometry of Microstructured Materials, H. T. Davis and J. C. C. Nitsche, eds., IMA vol. in Math. and its Appl.)

[72] G. Thomsen, Über konforme Geometrie. I, Grundlagen der konformen Flächentheorie, Abh. Math. Sem. Univ. Hamburg 3, 31-56 (1924)

[73] J. L. Weiner, On a problem of Chen, Willmore, et al., Indiana Univ. Math. J. 27, 19-35 (1978)

[74] J. H. White, A global invariant of conformal mappings in space, Proc. Amer. Math. Soc. 38, 162$164(1973)$

[75] N. M. Wigley, Mixed boundary problems in plane domains with corners, Math. Z. 115, 33-52 (1970)

[76] N. M. Wigley, Schauder estimates in domains with corners, Arch. Rational Mech. Anal. 104, 271$276(1988)$

[77] T. J. Willmore, Total curvature in Riemannian geometry, Ellis Horwood Ltd., Chichester, 1982 